\title{
Proceedings of the Association of British Neurologists, Birmingham, April 1991
}

CYSTEINE METABOLISM IN MOTOR NEURON DISEASE PATIENTS AND THEIR FAMILIES

SG Sturman, L Klorvza, MT Heafield, G Steventon, RH Wareing, AC Williams. Birmingham

Excitatory amino acids may have a role in the pathogenesis of motor neuron disease (MND). The neurotoxic sulphur amino acid cysteine is oxidised in vivo by the enzyme cysteine dioxygenase. This enzyme is inherited polymorphically and "extensive" and "poor" sulphoxidisers can be identified. We have previously reported a marked excess of "poor sulphoxidisers" among patients with MND. We have therefore measured plasma cysteine in MND patients and also sulphate, the ultimate product of its oxidation, using colorimetric methods. Blood was taken from fasted patients with MND $(\mathrm{N}=55)$ and healthy age and sex matched volunteers $(N=59)$. Cysteine levels are markedly elevated in MND $(0.602 \mathrm{nmol} / \mathrm{ml} / \mathrm{mg}$ protein, $\mathrm{SD}=0.307$ ) compared with controls $(0.373 \mathrm{nmol} / \mathrm{ml} / \mathrm{mg}$ protein, $S D=0.0224)$ ( $<<0.0001$, Mann-Whitney), and sulphate is very reduced $(1.38 \mathrm{pmol} / \mathrm{ml} / \mathrm{mg}$ protein) compared with controls $(4.41 \mathrm{pmol} / \mathrm{ml} / \mathrm{mg}$ protein) ( $p<0.0001$, Mann-Whitney). First degree relatives of MND patients also show an excess of "poor sulphoxidisers" (69\%, $\mathrm{N}=36$ ) compared with controls (35\%, $N=200$ ), however, their cysteine and sulphate levels are similar to controls. These results suggest that whilst $M N D$ is associated with "poor sulphoxidation" other factors disturb sulphur amino acid metabolism in these patients with potentially neurotoxic results.

AN AUTORADIOGRAPHIC STUDY OF N-METHYL-D-ASPARTATE (NMDA) RECEPTORS IN THE NORMAL HUMAN MOTOR SYSTEM AND MOTOR NEURON DISEASE

PJ Shaw, PG Ince, $M$ Johnson, D Bates, NEF Cartlidge. Newcastle upon Tyne

The amino acid glutamate is a major excitatory neurotransmitter in the human CNS and is the putative excitatory transmitter in the corticospinal tract and spinal cord interneuronal pathways. The actions of glutamate are mediated by several receptor subtypes of which the $\mathrm{N}$-methyl-D-aspartate receptor is the best characterised. There is evidence that abnormal stimulation of NMDA receptors may be important in the pathogenesis of several neurodegenerative disorders including motor neuron disease (MND).

We have determined the distribution and density of NMDA receptors in the motor system in post-mortem tissue from six neurologically normal controls, six cases of MND and six disease controls. NMDA receptors have been labelled using the non-competitive channel blocking agent $[3 \mathrm{H}]-\mathrm{MK}-801$ and the autoradiographs quantified with a computerised image analysis system. In the normal motor system NMDA receptors have been found: throughout the spinal grey matter with a high density in the substantia gelatinosa; in selective nuclei within the brainstem; and in all layers of the motor cortex with the greater density in laminae I-III. Focal areas of high binding were found in a distribution corresponding to lower motor neuron cell bodies. Substantial differences in receptors have been found in MND cases, correlating with neuropathological severity.

The anatomical distribution of NMDA receptors in relation to motor neuron somata may have important implications in selective vulnerability to excitotoxic injury.

CLINICAL APPLICATION OF A DNA PROBE TO FACIOSCAPULOHUMERAL MUSCULAR DYSTROPHY PW Lunt, M Upadhyaya, W Broadhead, M Sarfarazi, PS Harper. Bristol and Cardiff

Facioscapulohumeral muscular dystrophy (FSHD) is inherited as an autosomal dominant condition; age at onset and severity can vary widely. Possible genetic heterogeneity, particularly the proposal of "FSH-type spinal muscular atrophy (FSHSMA)" remains controversial. In a clinical and molecular genetic study of 38 FSHD families (175 affected subjects) we found no significant clinical evidence for gentic heterogeneity from presentation, onset age (mean 11.5 years), penetrance $(95 \%$ by 20 years), creatine kinase, or severity (at $40+$ years: $19 \%$ require wheelchairs, $30 \%$ remain presymptomatic).

Molecular genetic studies in 25 families from this panel have shown a consistent very close linkage of the disease gene to DNA probe D4S 139, mapping to the distal long arm of chromosome 4 (lod score $Z=38.4$ at recombination fraction theta $=0.01$ ). All 20 informative families, including three in which some subjects had previously been diagnosed as FSHSMA, and two families which define the opposite ends of a continuous clinical spectrum, concord with a single FSHD gene locus. The presumed genetic homogeneity, the very close linkage, and the usefulness of the hypervariable DNA probe D4S139 now make possible predictive, pre-natal and presymptomatic testing in families with FSHD. This already provides support for our previous clinical scoring of two disputed cases.

THE VALUE OF AN OCCUPATIONAL THERAPIST IN A NEUROLOGY CLINIC

V Patterson, E Kinnin. Belfast

Patients with physical disability are often seen at neurology clinics. They are usually seen by a doctor alone, and they may or may not be referred to other paramedical services. Doctors are expert at dealing with diseases and impairments, but usually are not expert at reducing disability and handicap, which are more the province of an occupational therapist (OT). Referral between the neurologist and community OT is haphazard and so we arranged for all disabled patients, attending the clinics of two neurologists, to be seen by a neurologically-trained occupational therapist at the clinic. A full evaluation of activities of daily living was performed, and actions taken under five headings; immediate advice, provision of equipment, treatment, referral to community OTs and referral to other agencies. After three months a questionnaire was sent to each patient asking for their evaluation of the actions taken. Thirty five per cent of patients seen required some action and a total of 129 actions were carried out, 33 for immediate advice, two for treatment, eight for provision of equipment, 51 referrals to the community OT and 35 referrals to other agencies. Over $50 \%$ of patients found the actions to be considerably helpful, and $80 \%$ expressed a wish to be seen by an OT at further clinics. A neurologicallytrained OT can make a significant contribution to many disabled patients who attend neurology clinics.

DEVELOPMENT OF A PROGNOSTIC INDEX FOR USE IN A TRIAL OF MEDICAL AND SURGICAL MANAGEMENT OF PRIMARY INTRACEREBRAL TUMOURS

JL Hutton, DF Smith, D Sandeman, PM Foy, MDM Shaw, IR Williams, DW Chadwick. Liverpool

The prognosis of intrinsic supratentorial tumours is variable. It is not known whether conservative or early aggressive (surgery and radiotherapy) is better for those tumours with a favourable prognosis. This dilemma can only be answered prospectively and would require the identification, at presentation, of patients with a good prognosis.

A retrospective analysis of 560 patients with intrinsic supratentorial tumours has been performed. Proportional hazards models for survival were derived using a stepwise selection procedure with only clinical and CT features as possible explanatory variables. A score indicating the prognosis was derived from the coefficients of the signs and symptoms, and survival rates of different prognostic groups was determined. Crossvalidation procedures indicated that the score was not dependent on a specific data set.

The variables of prognostic importance were age, first symptom of epilepsy, focal signs at presentation, a cystic lesion on CT scan and duration of symptoms prior to presentation. The model defined a group with a good prognosis (score $\leqslant /=9$, $\mathrm{N}=211$ ) and a group with a poor prognosis (score $>9, \mathrm{~N}=344$ ). The median survival was 27 months for those with a score of nine or less and three months for those with a score of greater than nine. An alternative score, derived from a model not including duration of symptoms, is also capable of defining groups with long (score $\leqslant /=16$, $\mathrm{N}=234$ ) and short (score $>16, \mathrm{~N}=325$ ) survival.

The model may provide a means of stratification of patients for inclusion in prospective randomised studies comparing aggressive and conservative management policies. 
SUBCORTICAL DEMENTIA IN CHRONIC TEMPORAL LOBE EPILEPSY

$S$ Brown, K O'Driscoll, M Vaughan, JS Snowden, NA Pearson, D Neary. Manchester

Fourteen adults with chronic temporal lobe epilepsy starting in childhood and of normal intelligence and educational attainments, developed progressive mental decline inexplicable by recurrent head trauma, hypoxia or excessive medication.

Seven males shared a common neuropsychological syndrome with profound slowness of cognition, concreteness of thinking, inefficient memorising and marked failure on "frontal lobe" tasks. SPECT scans in six revealed reduced tracer uptake in the anterior sub cortex.

Seven females displayed a characteristic neuropsychological profile with disordered thinking, paranoia and modest failure on frontal lobe tasks. In three, anterior subcortical tracer reductions were displayed on SPECT scan.

Recurrent seizure activity appears to disrupt functionally and structurally temporo frontal subcortical connections.

It is assumed that the sex influence on syndrome type relates to social and referral factors rather than biological processes.

The syndromes differ from that of dementia of frontal lobe type and therefore reflect subortical rather than frontal cortical disorder.

DELAYED DIAGNOSIS OF JUVENILE MYOCLONIC EPILEPSY

RA Grünwald, E Chroni, CP Panayiotopoulus. London

Fifteen patients with Juvenile Myoclonic Epilepsy (JME) were identified from a total of 180 patients referred to a new epilepsy clinic at St Thomas' Hospital during the period April 1989 to December 1990 (prevalence of $8.3 \%$ ). Of these, only one was referred with a putative diagnosis of JME. Diagnosis of the other patients on referral included "epilepsy", "grand mal", "petit mal", "photoconvulsive epilepsy" and "temporal lobe epilepsy". At least ten of the fifteen patients had been seen by an experienced neurologist in the United Kingdom before referral. Definitive diagnosis was delayed by a mean of 10.8 years. In seven patients inappropriate anticonvulsants had been prescribed resulting in poor seizure control. In four patients appropriate medication was withdrawn, two of these withdrawals being attempted because of prolonged absence of seizure. In fourteen of the fifteen patients response to appropriate medication at optimal dose was superior to previously prescribed drug regimens.

Factors delaying diagnosis of JME included lack of familiarity with the syndrome, failure to elicit a history of myoclonic jerking and misinterpretation of the characteristic EEG record which can show focal abnormalities. Delayed diagnosis of JME in the UK results in considerable avoidable morbidity among patients with epilepsy.

\section{QUANTIFICATION OF SEIZURE ACTIVITY}

JS Duncan, JWAS Sander. London

Seizure severity has been largely neglected in studies of epilepsy. To meet this need, we have developed a readily utilised seizure severity scale. Open interview of 50 patients with epilepsy and their relatives led to a list of 11 important factors: loss of awareness, warning, dropping of held object, fall, injury, incontinence, automatisms, convulsion, duration of seizure, time to return to normal and diurnal rhythm. These factors were combined into a scale, and weightings adjusted after repeated pilot studies. The scale was validated by comparison of scores with the opinions of patients, relatives and professionals of relative seizure severity in 24 inpatients, and in 37 patients who each had more than one seizure type. In these 37 patients, the mean relative severity score of each patient's seizures, obtained by the scale was 8.9 , compared with the mean patients' perceived relative severity of 8.5 . The mean difference between the ratios was $0 \cdot 6$. Reliability was reasonable. Inter-rater reliability $(n=93)$ the mean difference between the two observers was $1 \cdot 1$, coefficient of reliability $=13 \cdot 4$. Test-retest reliability $(n=101)$; the mean difference between the two sessions was -0.05 , coefficient of reliability $=15.9$. This seizure severity scale will be useful in the evaluation of medical and surgical treatment.

AN EPIDEMIOLOGICAL STUDY OF WILSON'S DISEASE IN IRELAND

M Reilly, M Hutchinson. Dublin

The worldwide prevalence of Wilson's Disease (WD) is estimated to be approximately 30 cases/million population. This widely accepted figure is based mainly on the work of Bachmann in East Germany and Saito in Japan. Both studies were based on specialis clinics dealing with Wilson's Disease and were inherently biased.

Because of the absence of any accurately based population study, the expectancy that an autosomal recessive condition should no have a uniform worldwide prevalence and the relative rarity of the condition in the experience of most clinical neurologists, we undertook an epidemiological study of WD in the Republic of Ireland over a 20 year period, 1970-89. Cases were ascertained from a register of all hospital admissions, all general physicians, neurologists, gastroenterologists, paediatricians, pathologists and death certificates.

Twenty five cases of WD were ascertained, an average prevalence of $2 \cdot 44 /$ million ( $1 \cdot 7 /$ million $1970-79,3 \cdot 6 /$ million $1980-89)$. Of 23 cases with full clinical details, seven presented with hepatic symptoms, five neurological, two psychiatric, one with other symptoms and the remainder through sibling screening. The average age at diagnosis was 18.8 years, the duration of symptoms varied between three months to six years before dignosis. Ten siblings were detected on screening families.

Even allowing for under-ascertainment we conclude that Wilson's Disease may not have a uniform worldwide prevalence and widely quoted prevalence figures should be viewed with caution.

THE PROGRAMMING OF SPEECH MOVEMENTS IN PARKINSON'S DISEASE

J Downes, K White, HJ Sagar, J Boucher. Sheffield

Studies of Parkinson's disease (PD) suggest that the basal ganglia play a critical role in the preparation of a complex motor act by integration of individual limb movements into a coordinated motor plan before movement (motor programming). However, it remains unclear whether deficits in motor planning also contribute to the abnormality of speech, in which discrete movements are less readily recognised. We examined the motor programming of speech in 20 early medicated patients with PD, none of whom was severely dysarthric, and 14 normal controls. Subjects were presented with strings of two to six letters (utterances), and required to read them aloud. Speech onset latency was the measure of motor programming for speech which, in normal subjects, is related to utterance length. Overall, the PD group was slower than the normal group but showed equivalent gain in performance when information of the stimuli was available prior to presentation. Both groups showed a linear increase in speech onset latency as utterance length increased but the slope for the PD group was steeper. These results suggest a role for the basal ganglia in the generation of a sequence of intact, discrete motor components of speech, which is distinct from speech preparation.

BRADYPHRENIA IN PARKINSON'S DISEASE

JA Cooper, HJ Sagar, P Tidswell, N Jordan. Sheffield

Studies in Parkinson's disease (PD) have shown selective deficits in simple reaction time (SRT) relative to choice reaction time (CRT). The critical difference between these tasks is that movement can be prepared in advance in SRT but not CRT; thus the abnormality in PD has been explained as a deficit in motor preprogramming or an attention-demanding process that precedes movement. However, most studies have not clearly separated the motor and cognitive aspects of performance. Other studies suggested that patients show slow but accurate cognitive performance (bradyphrenia) which may be pathophysiologically linked to depression. We examined RT under conditions of increasing cognitive complexity in $100 \mathrm{PD}$ patients and controlled for motor disability and depression. Subjects were presented with a series of visual and auditory stimuli of graded complexity and were required to respond by finger tap to specific aspects of the stimuli. RT of PD patients became progressively more abnormal with increasing cognitive complexity. The deficits were present in untreated, de novo nondepressed patients and were not affected by medication. Chronic disability and depression affected SRT but not CRT. These results show cognitive slowing in PD, independent of treatment or disease duration, which is distinct from motor slowing and depression.

VISUALLY INDUCED ILLUSION TILT IN PARKINSONS DISEASE

AP Moore, A Bronstein. London and Liverpool

Motor function in Parkinson's disease (PD) is often critically dependent upon visua information. The following experiment was carried out to investigate whether such visual dependence is a primary component of the disease or whether it compensates for primary deficiencies in the motor system.

Eleven patients and 10 age matched normal controls were required to set a straight line to the gravitational vertical (subject 
visual vertical"), 1) against a static visual background and 2 ) during rotation of a large disc ( $+/-72^{\circ}$ of visual angle) around the visual axis, at constant velocities between 20 and $60^{\circ} \mathrm{s}^{-1}$. There were no time constraints for this task so that performance was determined by the subject's perception of verticality. In all subjects the rotating disc induced a powerful illusion of self-rotation and tilt contralateral to the direction of disc rotation. Tilt was quantitated by the error in setting the straight line to vertical. With background, rotation at $20^{\circ} \mathrm{s}^{-1}$ the mean (SE) illusion for $\mathrm{PD}$, patients was $15 \cdot 8^{\circ}(1 \cdot 48)$ and for normal controls was $11.3^{\circ}(1 \cdot 3)$ : this difference was statistically significant $(p=0.022,95 \% \mathrm{CI}$ 0.7 to 8.3 ). The findings indicate that the abnormal visual dependence in PD is present at a perceptual level and therefore suggest that it is not secondary to the motor abnormality.

FAMILIAL PAROXYSMAL ATAXIA REPORT OF A FAMILY $\mathrm{CH}$ Hawkes. Ipswich

Three cases from one kindred who suffer from familial paroxysmal ataxia are described.

The index case, a woman aged 30 , described attacks for 25 years which were undiagnosed until very recently. Two of her sons, aged 12 and six years, were then also found to have the same condition, having been thought to suffer epilepsy. All three responded dramatically to acetazolamide.

Familial paroxysmal ataxia is a rare benign non-progressive disorder of childhood onset, characterised by bouts of ataxia with abrupt onset lasting minutes or hours. Cases may be identified on the basis of a suggestive history, nystagmus persisting between episodes and dominant inheritance. Atrophy of the cerebellar vermis is sometimes found and treatment with acetazolamide is often dramatically effective. This family is the first to be described in the UK but it is likely that many more exist, mislabelled as epilepsy or migraine.

GENETIC LINKAGE OF FAMILIAL ALZHEIMER'S DISEASE TO THE AMYLOID PRECURSOR PROTEIN LOCUS ON CHROMOSOME 21

J Brown, A Goate, MC Chartier, M Mullan, J Hardy, M Rosso. London

Linkage studies from several groups have suggested that the disease locus in familial Alzheimer's disease maps to chromosome 21. The gene for the amyloid precursor protein (APP), the most obvious candidate gene, also maps to the same region of chromosome 21 .

However there have been reports of recombinants between the disease locus and the APP gene-apparently excluding the APP gene as the site of the mutation causing the disease. Recently there has been increasing evidence for genetic heterogeneity in familial Alzheimer's disease, therefore only linkage analysis within single pedigrees is reliable. We present data from our most informative pedigree which show linkage to the APP locus. Sequencing the APP gene in this family has revealed a missense variant in exon 17 which results in a conservative change in the amino acid sequence from valine to isoleucine. This could be the cause of Alzheimer's disease in this family.
DIAGNOSTIC AND PRE-SYMPTOMATIC TESTING IN PRION DISEASES

J Collinge, MN Rossor, $M$ Poulter, F Owen TJ Crow, AE Harding. London and Manchester

Although the spongiform encephalopathies, Gerstmann-Sträussler syndrome (GSS) and Creutzfeldt-Jakob disease (CJD) are generally considered to be transmissible or infectious conditions, GSS is usually familial and CJD is familial in $15 \%$ of cases. Genetic linkage studies have demonstrated that these are genetic disorders with autosomal dominant inheritance linked to the prion protein $(\mathrm{PrP})$ gene on chromosome 20p. Molecular studies of the PrP gene in familial CJD and GSS have revealed several gene variants which alter the amino acid sequence of PrP and which occur only in affected or at-risk individuals. These include a 144 base pair insertion and point mutations at codons 102,117 and 200 . It is likely that these changes are pathogenic mutations, and the occurrence of one of these mutations in an affected individual from a family with a dementing/ataxic illnes or in apparently sporadic cases enables a molecular diagnosis of CJD/GSS or "prion disease" to be made. This represents the first primary neurodegenerative disorder in which a gene test is now available. It is already clear that the phenotype of this disease (defined at the DNA level) is more varied than previously appreciated both at the clinical and neuropathological level. PrP gene abnormalities have been identified in seven individuals who were not suspected to have spongiform encephalopathies on clinical grounds. Clearly pre-symptomatic testing can now be offered to at-risk individuals from families with identified PrP gene mutations, and three such tests have been completed at the National Hospital

DNA-BASED DIAGNOSIS OF MITOCHONDRIAL ENCEPHALOPATHY

\section{SR Hammans, AE Harding. London}

A point mutation in mitochondrial DNA (in the lysine transfer RNA gene) has recently been described in patients with the syndrome of myoclonic epilepsy and ragged red fibres (MERRF), a mitochondrial encephalomyopathy phenotype. The mutation can be detected rapidly by amplifying DNA using the polymerase chain reaction and subsequent restriction endonuclease digestion. Using this method we have analysed DNA derived from blood or muscle from 63 normal or disease control subjects, 30 patients with progressive myoclonic epilepsy or progressive myoclonc ataxia (of whom five were known to have RRF), and 71 encephalopathies.

The tRNA lysine mitochondrial DNA mutation was present in five out of seven patients with MERRF phenotype, but none of the patients with other mitochondrial myopathy syndromes or control subjects. Of particular interest is the observation of this mutation in a patient with progressive myoclonic ataxia who later developed bulbar dysfunction and had the pathological features of Leigh's syndrome, and one patient with myoclonic epilepsy but no other evidence of mitochondrial disease on clinical grounds. In all five patients the mutation was observed in DNA extracted from blood. Detection of this mutation is a rapid, non-invasive and inexpensive means of investigating patients with myoclonic epilepsy for mitochondrial disease.
BILATERAL PARAMEDIAN THALAMIC INFARCTION: A DISTINCT BUT POORLY RECOGNISED STROKE SYNDROME

M Reily, S Connolly, M Hutchinson. Dublin

Bilateral paramedian thalamic infarction is a well defined but seldom recognised clinical stroke syndrome. The typical clinical picture of sudden onset, vertical gaze palsy, somnolence and Korsokffian amnesia is due to infarction in the territory of the paramedian thalamic arteries when they arise from a common trunk from the posterior cerebral artery on one side. The advent of CT scanning increased recognition of this syndrome and by 1987, 40 cases had been reported.

Since 1987 we have seen six cases with classic clinical features; in four diagnosis was confirmed by CT scan and in two by MRI scan. All six cases, age range 19-87 years, presented with somnolence, five had vertical gaze palsy, two had Korsakoffian amnesia, and in addition bilateral third nerve palsies, eyelid retraction, bilateral ptosis and absent convergence were noted in some cases. All patients improved but only two have made complete recoveries.

This distinct clinical syndrome is probably underdiagnosed and, when suspected, can be readily confirmed by MRI scanning.

AETIOLOGY OF MULTIPLE SCLEROSIS: THE ASIAN, CARIBBEAN AND MEDITERRANEAN EVIDENCE

G Dean, M Elian. Medico-Social Research Board, Ireland, Charing Cross and Oldchurch Hospitals, England

The nature-nurture controversy regarding the aetiology of multiple sclerosis (MS) continues. We studied MS among immigrants to England. Asian immigrants had a very low risk of developing MS and Caribbean immigrants had a low risk, but not as low as the Asians. HLA studies in the Caribbean immigrants did not explain the low risk. The hospitalised prevalence among Italian immigrants was much the same as occurred in England and Wales, but no Maltese were found with MS in Greater London or the West Midlands although ten was the expected number. Studies in Sicily and Malta confirm that there was a relatively high prevalence in Sicily but a low prevalence in Malta. HLA studies failed to explain the ten fold difference. Immigrants to England from low MS prevalence areas of the world keep their low prevalence but their children born in the United Kingdom have a similar prevalence to that occurring in the general English population, strong evidence of an environmental influence perhaps in the genetically predisposed.

"CAMBS": A NEW RATING SCALE FOR MULTIPLE SCLEROSIS

CJ Mumford, MB Fraser, DAS Compston. Cambridge

The Cambridge Multiple Sclerosis Basic Score (CAMBS) represents a new rating scale which records current status in the context of the clinical course. CAMBS describes the impact of multiple sclerosis, distinguishing disability from handicap, and indicates the independent contribution of relapse and progression to current clinical activity. A single digit score of between 0 and 5 is assigned to each of these indices, giving a 
simple analysis for each encounter, for example, Disability 3, Relapse 1, Progression 2, Handicap 1. No attempt is made to integrate these variables, each of which may change independently with time.

The scale has been applied both to in- and out-patients $(n=183$ ) with demyelinating disease attending the neurology department at Addenbrooke's Hospital. Serial application of the scale is simple and informative both in registering the natural history of the disease and in recording the response to treatment. There is good reproducibility between separate examiners. The scale can be applied in less than one minute, and since it does not depend on physical examination can easily be used by para-medical staff. Patients welcome the opportunity of contributing to the assessment of handicap, and this provides additional continuity between examiners. For all these reasons CAMBS has important advantages over existing rating scales.

SEIZURES DUE TO MULTIPLE SCLEROSIS AJ Thompson, AG Kermode, DG MacManus, SG Moore, DH Miller, IF Moseley, DPE Kingsley, BE Kendall, WI McDonald. London

The reason for increased frequency of epilepsy in patients with multiple sclerosis (MS) is unknown. MRI readily demonstrates lesions in the white matter in MS and using the contrast agent Gadolinium-DTPA new lesions, including those involved in the cortex, are identified. We report the MRI results of seven patients with clinically definite MS who had generalised seizures during the course of their illness. In five patients this was associated with either the development of other neurological symptoms or a deterioration in existing ones. All EEGs showed epileptic discharges. MRI scans were carried out at an interval of between one day and two months following the onset of seizures and demonstrated cortical lesions in two patients and subcortical in the remaining five. Five patients were scanned within three weeks of their seizures and all demonstrated new disease activity. Serial scanning of one patient showed that improvement on MRI coincided with cessation of seizures and normalisation of the EEG.

This study is evidence that MS plaques may cause generalised seizures and that the areas involved tend to be either cortical or sub-cortical.

TEMPORARY REVERSAL OF BLOOD-BRAIN BARRIER ABNORMALITIES IN MULTIPLE SCLEROSIS BY HIGH DOSE STEROID THERAPY

DH Miller, IF Moseley, BE Kendall, DG MacManus, S Moore, AJ Thompson, WI McDonald. London

In multiple sclerosis (MS), the duration of disability in a relapse is shortened by high dose intravenous methylprednisolone (IVMP). The mechanism of benefit from IVMP is unclear; although one factor may be a general reduction in brain water. In patients with relapsing-remitting $M S$, new MRI lesions consistently display GadoliniumDTPA (Gd-DTPA) enhancement, indicating impairment of the blood-brain barrier (BBB). We report the serial Gd-DTPA enhanced MRI findings in MS patients being treated with IVMP.

Six MS patients were given IVMP $1 \mathrm{~g} /$ day for three days to treat a recent exacerbation of their condition. Brain MRI was performed at $0.5 \mathrm{~T}$ immediately before the first dose of IVMP, and again at one, three and seven days later. On each occasion, a precontrast $\mathrm{T}_{2}$-weighted sequence was performed, as well as a $T_{1}$-weighted sequence after administration of Gd-DTPA $0.1 \mathrm{mmol} / \mathrm{kg}$.

Fifty six enhancing lesions were presented before starting treatment. There was subsequently a rapid decrease in enhancement, which was maximal after three days, at which time $28(50 \%)$ lesions no longer enhanced, 25 displayed a definite reduction in enhancement, and only three (5\%) displayed the same degree of enhancement as seen before IVMP. After seven days many lesions reenhanced, such that $17(30 \%)$ now showed the same degree of enhancement before IVMP, and only $16(29 \%)$ showed no enhancement. One lesion showed increased enhancement at day seven.

We conclude that IVMP often produces a rapid but temporary reduction of $\mathrm{BBB}$ abnormalities in active MS lesions. Future study should be directed to elucidating the mechanism by which IVMP reduces BBB abnormalities as this may lead to new therapies with a similar effect on the BBB, but without long term toxicity.

THE OLFACTORY EVOKED RESPONSE AND EVIDENCE OF DISORDERED OLFACTION IN MULTIPLE

SCLEROSIS

CH Hawkes, G Kobal, B Shephard. Ipswich

Until recently it has not been possible to obtain pure olfactory evoked response (OEP) due to contamination by trigeminal signals, either because stimuli applied to the nose caused variation in flow which inadvertently activated the trigeminal mechano-receptors or because "impure" odours were used which had trigeminal chemo-receptor effects. The recently designed Kobal-Obermayer-olfactometer overcomes both artifacts.

Both healthy controls and multiple sclerosis (MS) patients have been studied by OEP and simple smell identification tests "UPSIT" (University of Pennsylvania Smel Identification Test). In a series of 22 control patients, an OEP to $\mathrm{H}_{2} \mathrm{~S}(20 \mathrm{ppm}$ in nitrogen) was obtained maximally at the vertex (PZ). The mean peak latency to $\mathrm{N} 1$ and P2 was approximately $422 \mathrm{~ms} / 556 \mathrm{~ms}$ respectively, with an amplitude of 10-20 uv. Of a total $12 \mathrm{MS}$ patients who displayed a well defined OEP there was significant increase of latency in 4 and abnormally low scores in $16 / 37$ of those tested on the UPSIT. Contrary to some earlier suggestions, these observations prove that the olfactory pathways are often affected in this disease.

A QUANTITATIVE MRI STUDY OF THE T1 AND T2 RELAXATION TIMES IN HIPPOCAMPAL SCLEROSIS GD Jackson, JS Duncan, A Connelly, SJ Austin. London

Reliable criteria for the MRI diagnosis of hippocampal sclerosis have recently been reported. These are a unilateral small hippocampus with an increased T2 weighted signal arising from it. We studied 20 patients with intractable complex partial seizures of temporal lobe origin with quantitative $\mathrm{T} 1$ and $\mathrm{T} 2$ maps as well as routine MRI. Increased T2 weighted signal was found in the hippocampal grey matter in all cases of hippocampal sclerosis, as diagnosed by MRI. T signal change was also present, being seen as a decreased signal intensity on inversion recovery images, and confirmed by quantitative analysis. All cases which showed T1 and T2 signal changes could be diagnosed nonquantitatively, based on the presence of hippocampal atrophy and signal changes, seen on optimally oriented inversion recovery and spin echo sequences.

TUMOURS THAT MIMIC TRANSIENT CEREBRAI

ISCHAEMIA

RJ Coleman, J Bamford, on behalf of UK TIA Aspirin Study Group. Leeds

In the UK, about 25000 people each year will have their first transient ischaemic attack (TIA). It is recognised that a few of these patients will have an underlying structural lesion but the literature gives little guidance as to which patients should have CT scans performed. We have examined the clinical records of a group of patients with cerebral tumours who were thought by British neurologists to have had TIAs.

Between 1979 and 1985, 2248 patients were recruited into the UKTIA Aspirin Trial.

Eleven patients were subsequently withdrawn after the identification of a cerebral tumour which was thought to be responsible for the symptoms, and we are able to report the details from 10 of these. Five had a malignant glioma and five had a meningioma. The delay between dates of entry and withdrawal ranged from 1-5 (mean 2) months for the gliomas and 1-23 (mean 9) months for the meningiomas. The most common presentation (in 6 patients) was with episodic hemisensory disturbance. Diagnostic reappraisal was usually prompted by either increasingly frequent attacks or by a major epileptic seizure. CT scanning was not requested prior to randomisation in six patients and one patient failed to attend. Of the other three scans, two were reportedly normal and one showed a lesion thought to be an area of infarction.

CEREBRAL PERFUSION IN ACUTE ISCHAEMIC STROKE: TRANSCRANIAL DOPPLER MONITORING DG Grosset, IB Squire, AD Morris, KR Lees, I Bone, JL Reid. Glasgow

To identify perfusion patterns following stroke, transcranial Doppler (TCD) examination was performed daily for three days in 30 consecutive patients, admitted 35 minutes -48 hours (median 5.75 hours) after an acute ischaemic cerebrovascular event. Extracranial duplex grading of internal carotid artery (ICA) stenosis was mild/moderate $<60 \%$, and severe/critical $>60 \%$. New infarcts were seen on CT in 14 cases, and old infarcts in five cases. In the ICA supplying the affected hemisphere, there was $>60 \%$ stenosis in six cases, and occlusion in one case. Lesser degrees of stenosis were present ipsilaterally in 11 cases, contralaterally in 10 cases, and there was bilateral $>60 \%$ stenosis in one case. TCD findings were as follows: 1) Reduced middle cerebral artery velocities (MCAV) and increased anterior cerebral artery velocities in the affected hemisphere, resembling findings in established MCA disease five cases). MCAV subsequently increased, suggesting reperfusion, in two of these cases. 2) Absent signals in hemispheres with massive infarction, but signals present contralaterally (two cases). This pattern was easily distinguished from: 3) Absent intracranial signals (three elderly female patients) 
and 4) Reduced quality signals (four cases). 5) In one case with ICA occlusion, a standard collateral pattern was seen. The MCAV/ ACAV ratio (in 23 cases in which vessels were identified bilaterally) was low in affected hemispheres (median 0.95) and normal in contralateral hemispheres (median 1.2), but the difference was not significant. These encouraging preliminary findings suggest that patterns of perfusion in acute stroke can be identified by TCD. The technique is likely to be useful in monitoring effects of thrombolytic therapy.

NATURE OF COGNITIVE DEFICITS FOLLOWING ANEURYSM RUPTURE: INFLUENCE OF ANEURYSM SITE AND CLINICAL COMPLICATIONS.

P Tidswell, PS Dias, HJ Sagar, AR Mayes, RDE Battersby. Sheffield

Cognitive outcome of aneurysmal subarachnoid haemorrage (SAH) managed by surgery has not been rigorously assessed. Amnesia has been reported following anterior communicating artery aneurysm (ACoAA) rupture, but it remains unclear whether this is specific to aneurysm site and whether it is dependent on other clinical variables. Accordingly, we gave a battery of neuropsychological tests to a consecutive sample of 37 patients with SAH from ACoAA, posterior communicating artery or middle cerebral artery aneurysms who had undergone surgery under the care of one neurosurgeon. Perioperative clinical events were enumerated by retrospective review of the case notes. The group was impaired on measures of frontal-lobe function, face recognition and immediate verbal memory but the ACoAA group did not differ quantitatively or qualitatively from the others. Only one patient of the total group had completely intact frontal lobe function. Moreover, frontal lobe deficits and memory deficits occurred together in all but one patient. Peroperative clipping of vessels and postoperative neurological complications were the best predictors of poor cognitive outcome. These results show that global cognitive deficits are common following aneurysm rupture treated surgically but are independent of aneurysm site. Furthermore, outcome may be influenced by surgical factors.

OBSERVATIONS IN AN ACUTE STROKE UNIT: IMPLICATIONS FOR A TRIAL OF THROMBOLYTIC THERAPY

AD Morris, DG Grosset, IB Squire, KR Lees, I Bone, JK Reid. Glasgow

An Acute Stroke Unit was established in a central teaching hospital (serving a population of 250000 ) for the immediate admission of all patients with an acute neurological deficit. During the first six months, 120 patients (49 males, 71 females) aged 36-91 (mean 72) years were admitted. One hundred and eight patients $(90 \%)$ had clinical evidence of a new cerebral hemisphere event. One hundred and twelve (93\%) underwent CT scanning. Four patients (3\%) had nonneurological illness.

The potential for enrolment into a trial of thrombolytic therapy was examined. The median time from event to presentation was five hours (range 40 minutes -48 hours) $40.5 \%$ of patients would be excluded from thrombolytic therapy due to presentation later than six hours; a further $9 \%$ would be ineligible because timing was unclear. Twenty three per cent of patients were aged over 80 , and $9 \%$ would be excluded because of a potential bleeding tendency. Twelve and a half per cent of patients had CT evidence of intracranial haematoma, and $4 \%$ had a large infarct associated with oedema and/or midline shift. Seven and a half per cent had other major systemic disease, and $6 \%$ had severe hypertension (diastolic blood pressure over $120 \mathrm{mmHg}$ ). Twenty one per cent of patients had a history of previous stroke and had residual deficit. Considering all exclusion criteria, $17.5 \%$ would have been eligible for treatment; earlier admission would have increased this to $23 \%$.

This pilot study demonstrates that although $77-82.5 \%$ would be ineligible, we could nevertheless enrol 40 to 50 patients annually for a trial of thrombolytic therapy.

A NEW STANDARD AXIS FOR ROUTINE BRAIN MRI GD Jackson, JS Duncan, $S$ Austin, A Connelly, J Shepherd. London

The choice of imaging planes in routine radiographic practice has been dictated largely by technical considerations. In the case of $x$ ray $C T$, artifacts created by the petrous temporal bones resulted in the use of standard imaging planes parallel to the orbitomeatal (OM) line. Interpretation of neuroanatomy as it is represented in images obtained in this plane has therefore become established, and has continued to be used in standard brain MRI. Current MR technology allows imaging planes in any orientation. After consideration of neuroanatomical structures, and the consequences of partial volume effects on these structures we conclude that the optimal plane for routine brain imaging with MRI is not the OM line, but an oblique coronal plane which is orientated in the plane of the anterior border of the medulla, and an oblique axial plane, which is at $90^{\circ}$ to this and at $30^{\circ}$ to the OM line. Imaging in these planes provides improved anatomical definition and easier interpretation of the neuroanatomy.

CEREBRAL BLOOD FLOW IN MIGRAINE USING 99M-TC-HMPAO AND SPET: VISUAL AND COMPUTERISED IMAGE ANALYSIS

PTG Davies, TJ Steiner, J Bowler, DC Costa, BE Jones, R Jewkes, F Clifford Rose. London

High-resolution regional cerebral blood flow (rCBF) images can be obtained in migraine using the cerebral blood flow marker $99 \mathrm{~m}$ - Tc - hexamethylpropyleneamineoxime (99m-Tc-HMPAO) and SPET. Migraine sufferers have been studied during spontaneous acute attacks $(n=35: 20$ acute classical migraine six in aura, 14 in the headache phase; 15 acute common migraine) and between attacks $(n=22: 10$ classical, 12 common migraine). On visual analysis of our images, cortical changes have been confined almost exclusively to acute classical migraine attacks but their significance to migraine pathophysiology is uncertain. However, a new computerised analysis system may clarify the situation. Images can now be formatted to a standard brain shape and size corresponding to Talairach's atlas ("Analyze"). Common variables (for example, injected dose of $99 \mathrm{~m}$-Tc-HMPAO) can be removed using an analysis of variance and the remaining data subjected to pixcel by pixcel analysis for areas of significant change from normal with $p<0.001$ ("Promatlab"). This method of analysis has added a new dimension to the study of rCBF in migraine.

THE UK MOTOR NEURON DISEASE TWIN STUDY-A NEW TECHNIQUE AND PRELIMINARY RESULTS CH Hawkes, AJ Graham. Ipswich

The aetiology of motor neuron disease (MND) is still obscure, with no clear indication of the respective roles of genetic and environmental factors. A twin study is one of the most powerful methods for determining the relative importance of these aspects.

Unbiased and adequate collection of twin pairs is difficult, especially in MND which has a low prevalence (approx. 4: 100 000). Assuming a population of $4.8 \times 10^{6}$, and twin ratio $1: 80$, one would expect to find only 19 MND patients with a twin.

We have devised an alternative new approach, which involves tracing the co-twins of people who have died from MND in England and Wales over a ten year period (1970-88). This is achieved by obtaining delimited copies of all MND death certificates and using this information to search the Birth Register Indexes to identify possible twins. This method produced 120 twin pairs. Details of the co-twins are then sent to the National Health Service Central Register to trace their general practitioners (GP). A letter is sent to the GPs requesting access to the co-twins, and if given, the co-twins are approached about participating in the study.

An extensive interview questionnaire will be utilised that relates to both twins. Zygosity can be ascertained with $94 \%$ certainty by the "pea in a pod" question.

So far, our study has unearthed 80 like sex, 40 different sex twins and only two concordant like sex pairs.

DOMINANT DISTAL MYOPATHY WITH ABNORMAL NEUROMUSCULAR TRANSMISSIONS

BRF Lecky, B Tedman, PH Humphrey, JM Mackenzie. Liverpool

Four generations of an English family are affected by a myopathy showing weakness of face, neck, truncal and distal limb muscles. There are no neonatal or infantile symptoms. Affected individuals show weakness in childhood with or without progression in early adult life. Disability is variable. There is no response to anticholinesterase drugs but some response to $2: 4$ diaminopyridine.

Serum CK levels are normal and antiacetylcholine receptor antibody is absent. Muscle biopsy shows hypertrophic fibres and groups of rounded atrophic fibres with type I predominance. EMG shows clear myopathic features. Repetitive nerve stimulation studies are normal. SFEMG shows marked blocking and moderately increased jitter.

The condition differs clinically and electrophysiologically from other reported dominant distal myopathies. A post-synaptic myasthenic disorder is unlikely because of the lack of anticholinesterase-responsiveness and a pre-synaptic disorder is possible.

ASSESSMENT OF SWALLOWING IN NEUROLOGICAL PATIENTS-A PILOT STUDY

KM Nathadwarawala, I Graham, A McGroary, CM Wiles. Cardiff

Routine clinical examination may not reliably detect abnormal swallowing or aspiration. 
Swallowing and lower cranial nerve function were prospectively assessed in 81 neurological inpatients utilising a standard questionnaire, neurological examination and a timed test of drinking. Patients were categorised according to whether they perceived that they did or did not have a swallowing problem. Reliability of the methods was confirmed by testing 26 patients on two separate days.

Patients who said that their swallowing was abnormal (27, mean age 55.0 years, 12 males) had a swallowing speed of $3.76 \pm 3.61 \mathrm{ml} / \mathrm{s}^{-1}$ (mean, SD): those who denied a problem $(54$, mean age 47.8 years, 32 males) had a speed of $17 \cdot 14 \pm 12 \cdot 86 \mathrm{ml}$ $\mathrm{s}^{-1}(\mathrm{p}<0.002)$. A speed of $<10 \mathrm{ml} / \mathrm{s}^{-1}$ was strongly associated with the complaint of abnormal swallowing with a sensitivity of $96 \%$, specificity of $69 \%$ and one false negative result. The palatal and pharyngeal reflexes were an unreliable guide for abnormal swallowing. 12 of 17 patients who denied a problem but drank at $<10 \mathrm{ml} / \mathrm{s}^{-1}$ were thought to be at risk of aspiration, or to have a swallowing problem based on either questionnaire or examination.

VIGABATRIN AND EPILEPSY

JWAS Sander, YM Hart, SD Shorvon. Chalfont and London

We have treated over 250 patients with severe intractable epilepsy with vigabatrin, an irreversible inhibitor of GABA aminotransferase. Approximately one third of patients had improved seizure control. The commonest serious side effect was behavioural disturbance. There were 15 cases of pychosis following the introduction of vigabatrin. Ten cases had no previous history of psychosis. In nine cases psychosis occurred following a marked change in the habitual pattern of seizure activity; in four of these cases it developed after a period of seizure freedom followed by a cluster of seizures and in the other five cases the patients became seizure free and developed the psychosis during remission. In five patients there was no clear relationship to seizure pattern. Another patient developed psychosis after taking an overdose of between eight and $12 \mathrm{~g}$ of vigabatrin. The mean dose at onset of the psychosis was $2610 \mathrm{mg}$ (range: $500-4000 \mathrm{mg}$ ), and the period from initiation of therapy to the onset of psychosis varied from five days to 32 weeks. In all cases the psychosis resolved following cessation of vigabatrin. The mechanism of this behaviour change is unclear. We would urge caution in patients starting vigabatrin, particularly in the presence of a previous history of behavioural disturbances, and such patients should be carefully monitored.

AGE AND LOCALIZATION-RELATED IDIOPATHIC EPILEPSIES: A 17 YEAR CLINICAL AND ELECTROENCEPHALOGRAPHIC STUDY OF 94 PATIENTS

CP Panayiotopoulus. London

This is a clinical and electroencephalographic (EEG) study of 94 patients with "age and localization-related idiopathic epilepsies" (ALRIE) seen between 1973 and 1983. The majority of them, mainly those with benign childhood epilepsy with occipital paroxysms (BCEOP), have been followed up prospectively from 1973 to date. The prevalence of ALRIE was $26.6 \%$ amongst patients with onset of seizures between two to 13 years of age ( 354 children). Benign childhood epilepsy with centro-temporal spikes (BCECTS) was the commonest ( 55 boys and 17 girls). The main clinical manifestations consisted of infrequent, mainly nocturnal seizures with oro-pharyngeal movements, aphemia, partial motor and generalised convulsions. There were two peaks of the age of onset at seven and 10 years old. Prognosis was excellent. BCEOP was found in 18 children with a female preponderance (11 girls). The main clinical manifestations were infrequent, usually nocturnal seizures with vomiting, tonic deviation of the eyes often followed by hemi- and generalised convulsions. Prolonged ictal episodes, sometimes for hours were reported in eight of them. The mean age of onset was five years with complete remission usually within one to three years of onset. The EEG showed high amplitude centro-temporal spikes in BCECTS and occipital paroxysms in BCEOP. Another four children with infrequent seizures and a similarly benign course showed spikes consistently located in the frontal (two children) and midline electrodes (the remaining two) which indicates that benign childhood epilepsies with frontal and midline spikes, although rare, do also exist. The relation of all these benign focal epilepsies is indicated by the high incidence of multiple spike foci (CTS, OS, Frontal, Midline, Somatosensory evoked CTS) among children with ALRIE. 